OPEN ACCESS

Edited by:

Joav Merrick,

Ministry of Social Affairs, Israel

Reviewed by:

Carlo Pietrasanta,

Boston Children's Hospital and

Harvard Medical School,

United States

Cipta Pramana

Tarumanagara University, Indonesia

${ }^{*}$ Correspondence:

Yuan Wei

weiyuanbysy@163.com

Yangyu Zhao

zhaoyangyu@bjmu.edu.cn

Xiaoli Wang

xlwang@bjmu.edu.cn Jie Qiao

jie.qiao@263.net

tThese authors have contributed equally to this work and share first

authorship

Specialty section: This article was submitted to

Children and Health,

a section of the journal

Frontiers in Pediatrics

Received: 30 July 2021

Accepted: 14 October 2021

Published: 23 November 2021

Citation:

Wu T, Chen L, Wang Y, Shi H, Niu J, Yin $X$, Li M, Tan C, Jiang H, Zheng $D$,

Wei Y, Zhao Y, Wang $X$ and Qiao J

(2021) Effects of SARS-CoV-2 Infection During Late Pregnancy on

Early Childhood Development: A

Prospective Cohort Study.

Front. Pediatr. 9:750012.

doi: 10.3389/fped.2021.750012

\section{Effects of SARS-CoV-2 Infection During Late Pregnancy on Early Childhood Development: A Prospective Cohort Study}

\author{
Tianchen $\mathrm{Wu}^{1+}$, Lian Chen ${ }^{2,3,4 t}$, Yuanyuan Wang ${ }^{2,3,4 t}$, Huifeng Shi ${ }^{2,3,4}$, Jieqiong Niu ${ }^{1}$, \\ Xiaohan Yin ${ }^{1}$, Mengshi Li ${ }^{1}$, Chang Tan ${ }^{1}$, Hai Jiang ${ }^{2,3,4}$, Danni Zheng ${ }^{2,3,4}$, Yuan Wei ${ }^{2,3,4 *}$, \\ Yangyu Zhao ${ }^{2,3,4 *}$, Xiaoli Wang ${ }^{1,5 *}$ and Jie Qiao ${ }^{2,3,4 *}$ \\ ${ }^{1}$ Department of Maternal and Child Health, School of Public Health, Peking University, Beijing, China, ${ }^{2}$ Department of \\ Obstetrics and Gynecology, Peking University Third Hospital, Beijing, China, ${ }^{3}$ National Clinical Research Center for \\ Obstetrical and Gynecology, Beijing, China, ${ }^{4}$ National Center for Healthcare Quality Management in Obstetrics, Beijing, \\ China, ${ }^{5}$ Key Laboratory of Reproductive Health, National Health Commission, Beijing, China
}

Background: There is little direct or indirect evidence of the effects of severe acute respiratory syndrome coronavirus 2 (SARS-CoV-2) infection during pregnancy on early childhood development.

Methods: We conducted a prospective, observational cohort study in China from May 1 to October 31, 2020, that enrolled 135 mother-infant dyads: 57 dyads in the infection cohort and 78 in the non-infection cohort. Among all infants, 14.0\% were preterm birth in the infection cohort and $6.4 \%$ in the non-infection cohort. Participants were followed by telephone interviews to collect demographic characteristics, medical records of coronavirus disease 2019, breastfeeding data, and early childhood development was assessed by the Age and Stage Questionnaire (ASQ-3) and Age and Stage Questionnaire Social-Emotional (ASQ:SE-2) Chinese versions at 3 months after childbirth. We used multivariable Poisson regression models to estimate the relative risk (RR) of SARS-CoV-2 infection. Multivariable linear regression models and a mediation model were used to test the direct and indirect associations between SARS-CoV-2 infection and the ASQ-3 score. This study was approved by the Peking University Third Hospital Medical Science Research Ethics Committee (No. IRB00006761-M2020127).

Results: In the infection cohort, $13.6 \%$ of the children showed social-emotional developmental delay, and $13.5 \%$ showed overall developmental delay. The corresponding rates in the non-infection cohort were 23.4 and $8.1 \%$. Compared with the non-infection cohort, SARS-CoV-2 infection during pregnancy did not increase the risk of social-emotional $(R R=0.87,95 \%$ $\mathrm{Cl}$ : $0.51-1.49)$ or overall $(\mathrm{RR}=1.02,95 \% \mathrm{Cl}$ : 0.60-1.73) developmental delay. The mediation model showed that SARS-CoV-2 infection indirectly affected the ASQ-3 score by increasing the length of mother-infant separation. 
Conclusions: SARS-CoV-2 during late pregnancy did not increase the risk of developmental delay of the offspring 3 months after delivery. However, SARS-CoV2 may have indirect effects on early childhood development by increasing motherinfant separation.

Keywords: COVID-19, SASRS-CoV-2, pregnancy, early childhood development, mother-infant separation, mediation model

\section{INTRODUCTION}

The coronavirus disease 2019 (COVID-19) pandemic is the most serious global public health crisis of the 21st century. As of July 29, 2021, more than 190 million people worldwide have been infected with severe acute respiratory syndrome coronavirus 2 (SARS-CoV-2) (1). Coronavirus infection affects pregnant women more than the general population, and infected pregnant women are more likely to need intensive care treatment than non-pregnant women $(2,3)$. The most common reported adverse perinatal outcomes related to COVID-19 are preterm birth and cesarean delivery (3-5).

Another concern with COVID-19 infection during pregnancy is that of the potential effects on early childhood development (6), which might occur via two possible pathways. The first is the pathophysiological effects of SARS-CoV-2 infection. Although, there is insufficient evidence of vertical SARS-CoV-2 infection transmission (7), mothers infected during pregnancy have higher interleukin-6 (IL-6) levels than non-pregnant women (8). IL-6 is an indicator of maternal systemic inflammation that is potentially related to infant brain development (9) and inversely associated with offspring cognition at 12 months of age (10). The second pathway is mother-infant separation caused by hospitalization. In the early phase of the pandemic, due to insufficient understanding of the transmission dynamics of COVID-19, and to treat infected pregnant women promptly and protect their newborns, mothers were separated from their infants for long periods (11). Mother-infant separation in early childhood has adverse effects on child development (12). Unfortunately, there is no evidence of whether SARS-CoV-2 infection during pregnancy increases the risk of neurodevelopmental delay in early childhood.

Our team has reported the clinical characteristics of pregnant women infected with SARS-CoV-2 (in the third trimester, mainly in the late trimester) and assessed the early development of their children $(4,13)$ using the National Epidemic Reporting System (NERS) of the National Health Commission of China. In this study, we used the previous data as an infection cohort, and established a non-infection cohort in Wuhan, China, recruiting pregnant women and their infants who had never been infected

\footnotetext{
Abbreviations: COVID-19, coronavirus disease 2019; SARS-CoV-2, severe acute respiratory syndrome coronavirus 2; NERS, National Epidemic Reporting System; RT-PCR, real-time reverse transcription polymerase chain reaction; ASQ-3, Age \& Stage Questionnaire Chinese version; ASQ:SE-2, Age \& Stage Questionnaire Social-Emotional Chinese version; IQR, interquartile range; $\mathrm{RR}$, relative risk; NICU, neonatal intensive care unit; SARS-CoV, severe acute respiratory syndrome coronavirus; MERS-CoV, Middle East respiratory syndrome coronavirus.
}

with SARS-CoV-2, to compare the early childhood development of the two cohorts and explore the potential secondary effects of COVID-19 pandemic on early childhood development.

\section{MATERIALS AND METHODS Study Design and Participants}

This prospective, observational cohort study was conducted from May 1 to October 31, 2020. It established an infection cohort who were followed from May 1 to July 1, 2020, and a noninfected cohort who were followed from July 7 to October 31 , 2020. The infection cohort were retrieved from the NERS until April 30, 2020. The inclusion criteria of the infection cohort were (1) a confirmed case of COVID-19 defined as a suspected case (a person who had both the epidemiological history and clinical manifestations) with a positive result on high-throughput sequencing or real-time reverse transcription polymerase chain reaction (RT-PCR) assay of nasal and pharyngeal swab specimens (14), (2) a pregnant woman diagnosed with COVID-19, (3) the onset of COVID-19 was at any week of pregnancy, and (4) informed consent was obtained. Of the 138 cases retrieved from the NERS, 81 met the inclusion criteria, including 65 deliveries and 16 abortions. We included only the 65 deliveries of whom 57 (87.7\%) of the women completed all follow-up procedures (13) (Figure 1).

The non-infection cohort were recruited through an online assistance and consultation platform for pregnant women in Wuhan initiated by doctors during the COVID-19 pandemic. The inclusion criteria of the non-infection cohort were (1) a pregnant woman not diagnosed with COVID-19, (2) not a close contact or suspected case, or not quarantined, (3) a live birth without neonatal death, and (4) informed consent was obtained. Ninety-five cases met the inclusion criteria and 78 (82.1\%) completed all of the follow-up procedures (Figure 1). In the recruitment of participants, we did not limit the gestational age at delivery in both infection cohort and non-infection cohort. This study was approved by the Peking University Third Hospital Medical Science Research Ethics Committee (No. IRB00006761M2020127).

\section{Follow-Up Procedures}

For all participants in both cohorts, we conducted a followup at four time points. (1) The first was to obtain informed consent when the participant was recruited, and we collected the diagnosis, treatment, and outcomes of COVID-19 from medical records. (2) One week $\left(7^{+0 \sim 2}\right.$ days) after delivery, we collected data on hospitalized delivery, screening results of 


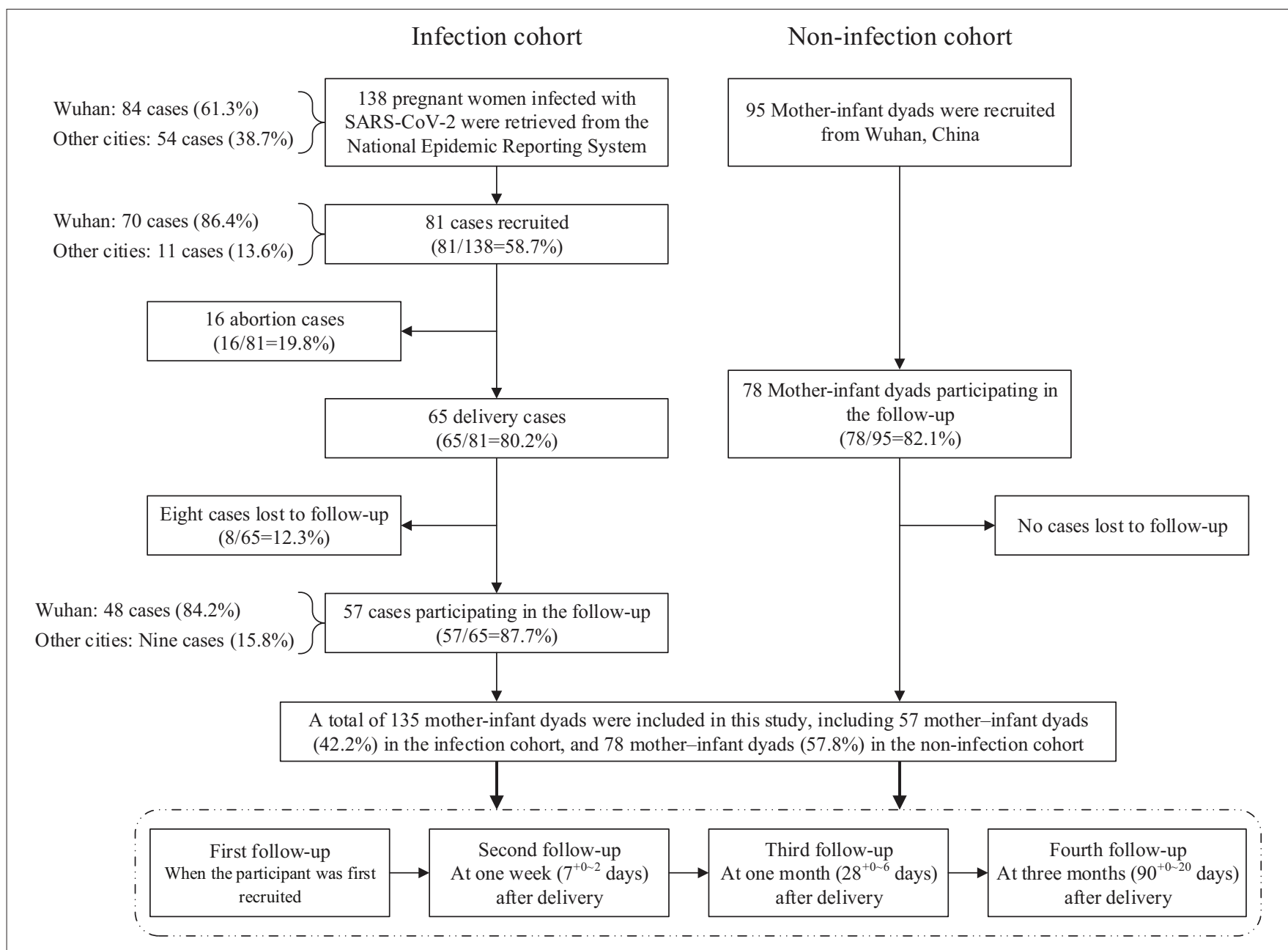

FIGURE 1 | Flowchart of the prospective cohort study.

SARS-CoV-2 for maternal and neonatal specimens (RT-PCR assay of neonatal throat swab, cord blood, amniotic fluid, breast milk, meconium, or placenta, and $\operatorname{IgG}$ and $\operatorname{IgM}$ in neonatal serum), postpartum care from medical records; the duration of quarantine, mother-infant separation, and neonatal feeding were collected by telephone interviews. (3) At 1 month $\left(28^{+0 \sim 6}\right.$ days), the durations of quarantine and mother-infant separation, and neonatal feeding were obtained by telephone interviews. (4) At 3 months $\left(90^{+0 \sim 20}\right.$ days), the durations of quarantine and mother-infant separation, and neonatal feeding were obtained by telephone interviews, and early childhood development was assessed by sending an online questionnaire to the mobile phones of the mothers. The breastfeeding data collected in our study referred to both exclusively and partial breastfeeding; we did not distinguish them. Mother-infant separation included two scenarios, the first followed national policy (11) requiring neonates delivered by infected pregnant women to be observed in an isolated observation ward for at least 14 days. The second was that after the termination of medical quarantine, some mothers chose to continue home quarantine, which extended the motherinfant separation time.

\section{Early Childhood Development Assessment}

Early childhood development was assessed using the Chinese versions of the Age and Stage Questionnaire (ASQ-3) and Age and Stage Questionnaire Social-Emotional (ASQ:SE-2). All infants were assessed at 3 months after childbirth. ASQ-3 consists of five domains: communication, gross motor, fine motor, problem solving, and personal-social. ASQ:SE-2 was used to assess social-emotional development. In this study, the mothers of the infants completed both ASQ-3 and ASQ:SE-2. A child whose ASQ-3 score was lower than the Chinese norm indicated developmental delay in that domain, and developmental delay in any ASQ-3 domain indicated overall developmental delay (15). A total ASQ:SE-2 score higher than the Chinese norm indicated social-emotional developmental delay (16).

\section{Statistical Analyses}

Continuous variables were described as the median and interquartile range (IQR); the two cohorts were compared using the Wilcoxon signed-rank test. Categorical variables were described as the frequency and percent, and the two cohorts were compared using the Chi-square test or Fisher's exact 
TABLE 1 | Demographic characteristics, perinatal outcomes, and early childhood development between infection cohort and non-infection cohort.

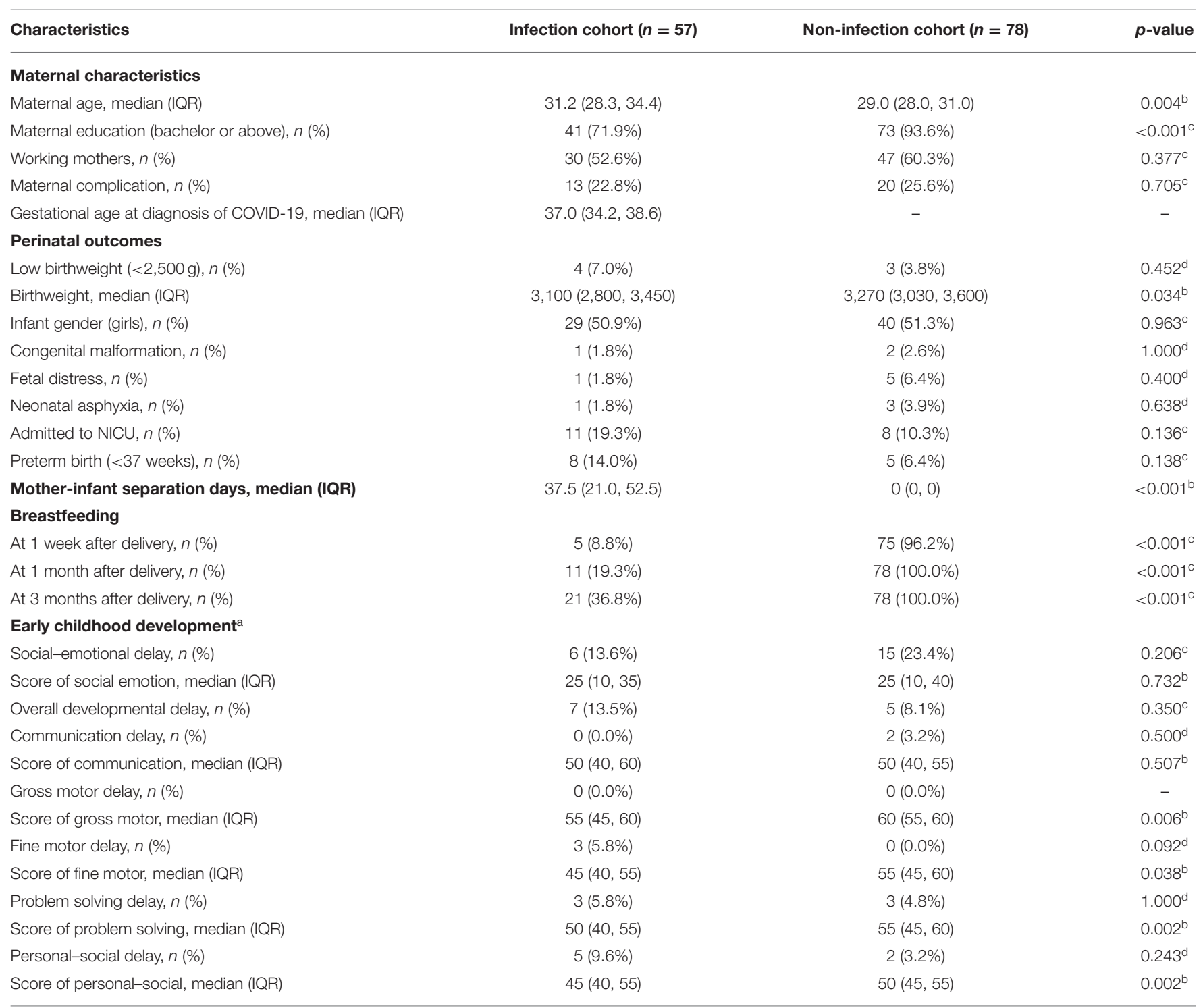

IQR, interquartile range; NICU, neonatal intensive care unit. a In the infection cohort, 13 participants did not respond to ASQ:SE-2, five participants did not respond to ASQ-3; In the non-infection cohort, 14 participants did not respond to ASQ:SE-2, 16 participants did not respond to ASQ-3. ${ }^{b}$ Wilcoxon signed-rank test. ${ }^{~ C}$ Chi-square test. ${ }^{d}$ Fisher's exact test.

test. We estimated the adjusted relative risk (RR) of SARSCoV-2 infection on developmental delay using multivariable Poisson regression models. The associations between SARS-CoV2 infection and the ASQ-3 and ASQ:SE-2 scores were tested using multivariable linear regression models. We adjusted for the length of mother-infant separation, low birthweight (yes vs. no), infant gender (boy vs. girl), preterm (yes vs. no), admitted to neonatal intensive care unit (NICU; yes vs. no), and breastfeeding at 3 months after delivery (yes vs. no) in the multivariable Poisson and linear regression models.

Based on the preliminary analysis, we hypothesized that the effects of SARS-CoV-2 infection on early childhood development may be mediated by mother-infant separation. Therefore, we fitted a mediation model to test the indirect effect. This mediation model included five paths: Path a represented the effect of SARSCoV-2 infection on the length of mother-infant separation; Path $\mathrm{b}$ represented the effect of length of mother-infant separation on gross motor development; Path $\mathrm{a} \times \mathrm{b}$ (indirect effect) represented the mediating effect of SARS-CoV-2 infection on gross motor development mediated by length of mother-infant separation; Path $c^{\prime}$ (direct effect) represented the remaining effect of SARSCoV-2 infection on gross motor development that could not be explained by the mediating effect in this model; and Path $\mathrm{c}$ (total effect) represented the total effect of SARS-CoV-2 infection on gross motor development.

Descriptive analyses and multivariable analyses were implemented using SAS ver. 9.4 (SAS Institute, Cary, NC, USA). The mediation model was implemented using the mediation 
TABLE 2 | Association between SARS-CoV-2 infection and early childhood development.

\begin{tabular}{|c|c|c|c|c|}
\hline Early childhood development & $\mathrm{RR}(95 \% \mathrm{Cl})^{\mathrm{a}}$ & $p$-value & $\beta(95 \% \mathrm{Cl})^{\mathrm{b}}$ & $p$-value \\
\hline \multicolumn{5}{|l|}{ Social emotion } \\
\hline SARS-CoV-2 infection & $0.87(0.51,1.49)$ & 0.617 & $2.94(-5.11,11.00)$ & 0.474 \\
\hline Mother-infant separation days & $1.00(0.99,1.01)$ & 0.906 & $-0.11(-0.27,0.04)$ & 0.154 \\
\hline \multicolumn{5}{|l|}{ Overall development ${ }^{\mathrm{c}}$} \\
\hline SARS-CoV-2 infection & $1.02(0.60,1.73)$ & 0.951 & - & - \\
\hline Mother-infant separation days & $1.00(0.99,1.01)$ & 0.963 & - & - \\
\hline \multicolumn{5}{|l|}{ Communication } \\
\hline SARS-CoV-2 infection & $1.01(0.58,1.75)$ & 0.968 & $0.76(-4.62,6.14)$ & 0.782 \\
\hline Mother-infant separation days & $1.00(0.99,1.01)$ & 0.898 & $-0.06(-0.17,0.04)$ & 0.252 \\
\hline \multicolumn{5}{|l|}{ Gross motor $^{d}$} \\
\hline SARS-CoV-2 infection & - & - & $-0.57(-4.05,2.92)$ & 0.751 \\
\hline Mother-infant separation days & - & - & $-0.09(-0.16,-0.02)$ & 0.008 \\
\hline \multicolumn{5}{|l|}{ Fine motor } \\
\hline SARS-CoV-2 infection & $1.04(0.60,1.81)$ & 0.887 & $-1.65(-7.18,3.88)$ & 0.559 \\
\hline Mother-infant separation days & $1.00(0.99,1.01)$ & 0.889 & $-0.08(-0.19,0.03)$ & 0.146 \\
\hline \multicolumn{5}{|l|}{ Problem solving } \\
\hline SARS-CoV-2 infection & $1.02(0.60,1.75)$ & 0.935 & $-0.86(-5.87,4.14)$ & 0.736 \\
\hline Mother-infant separation days & $1.00(0.99,1.01)$ & 0.970 & $-0.08(-0.18,0.02)$ & 0.111 \\
\hline \multicolumn{5}{|l|}{ Personal-social } \\
\hline SARS-CoV-2 infection & $1.01(0.59,1.74)$ & 0.973 & $-0.43(-6.87,6.02)$ & 0.897 \\
\hline Mother-infant separation days & $1.00(0.99,1.01)$ & 0.993 & $-0.02(-0.13,0.12)$ & 0.958 \\
\hline
\end{tabular}

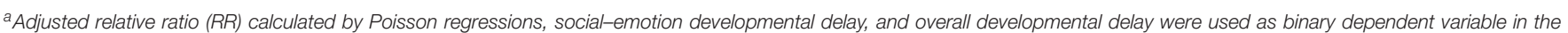

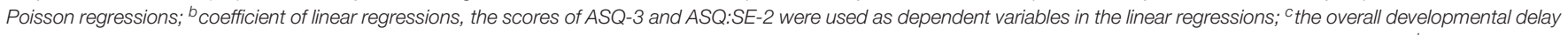

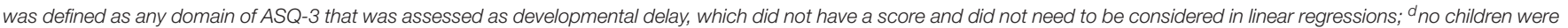

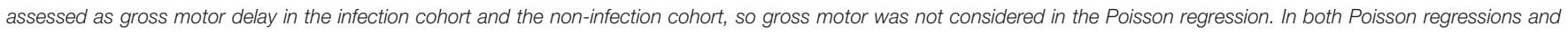

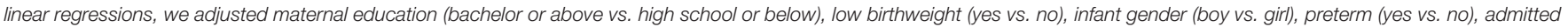
to NICU (yes vs. no), and breastfeeding at 3 months after birth (yes vs. no).

package PROCESS ver. 3.5 by Andrew F. Hayes for SPSS (17). All statistical tests were two-tailed, and $p<0.05$ was considered statistically significant.

\section{RESULTS}

\section{Demographic Characteristics and Main Outcomes}

Table 1 summarizes the demographic characteristics, perinatal conditions, and early childhood development. The median age of the mothers was significantly higher in the infection cohort. A significantly lower proportion of mothers in the infection cohort had a bachelor's degree or above. The proportions of low birthweight, congenital malformation, fetal distress, neonatal asphyxia, admitted to NICU, and preterm birth did not differ significantly between the two cohorts.

In the infection cohort, four pregnant women $(7.0 \%)$ were infected in the second trimester (19.5, IQR $=15.8-23.1$ weeks) and the other $53(93.0 \%)$ in the third trimester (37.4, IQR $=35.1-38.8$ weeks). Clinically, 49 (86.0\%) pregnant women were diagnosed as mild cases and eight (14.0\%) as severe cases. Furthermore, we collected screening results of RT-PCR assay of neonatal throat swab (51 cases), cord blood (3 cases), amniotic fluid ( 2 cases), breast milk (12 cases), meconium (3 cases), and placenta ( 2 cases). A single neonate had a positive RT-PCR throat swab $36 \mathrm{~h}$ after delivery, but was negative in subsequent tests, as previously reported $(13,18)$. There were 17 cases having a testing of IgG and IgM in neonatal serum: eight cases (47.1\%) with IgG $(+)$, including three cases $(17.6 \%)$ with $\operatorname{IgM}(+)(13)$.

From the date of birth, the separation time of mothers and infants in the infection cohort was significantly longer than that in the non-infection cohort. In the non-infection cohort, eight mothers were separated from their infants for a median of 19 (IQR $=7.0-36.0)$ days because their infants were admitted to the NICU. The proportions of breastfeeding (did not distinguish exclusively and partial breastfeeding) in the infection cohort at 1 week (8.8 vs. $96.2 \%), 1$ month (19.3 vs. $100.0 \%$ ), and 3 months (36.8 vs. $100.0 \%$ ) were significantly lower than in the non-infection cohort. Social-emotional developmental delay was seen in $13.6 \%$ of the children in the infection cohort and $23.4 \%$ of those in the non-infection cohort $(p>0.05)$. Similarly, overall developmental delay was detected in $13.5 \%$ of the children in the infection cohort and $8.1 \%$ in the non-infection cohort; the difference was also not significant. The proportions with developmental delay in communication, fine motor, problem solving, and personal-social did not differ significantly between the two cohorts. No children were assessed as having gross motor developmental delay in either cohort. However, the gross motor, fine motor, problem solving, and personal-social ASQ-3 scores were significantly lower in the infection cohort. 


\section{Association Between SARS-CoV-2 Infection and Early Childhood Development}

After adjusting for potential confounders, we found that SARSCoV-2 infection during pregnancy did not increase the risks of social-emotional (RR $=0.87,95 \%$ CI: $0.51-1.49$ ) and overall (RR $=1.02$, 95\% CI: 0.60-1.73, Table 2) developmental delay. SARSCoV-2 infection was also not significantly associated with the ASQ-3 and ASQ:SE-2 scores. Although we found that motherinfant separation did not increase the risks of social-emotional $(\mathrm{RR}=1.00,95 \% \mathrm{CI}: 0.99-1.01)$ and overall $(\mathrm{RR}=1.00,95 \%$ CI: 0.99-1.01, Table 2) developmental delay, multivariable linear regression models indicated that the length of mother-infant separation was significantly negatively correlated with the gross motor score ( $\beta=-0.09,95 \%$ CI: -0.16 to -0.02$)$.

\section{Mediation Effect Between SARS-CoV-2 Infection and Early Childhood Development}

Table 3 and Figure 2 show the components of the mediation model. SARS-CoV-2 infection had significant total (Path $c, p$ $=0.009)$ and indirect $($ Path $\mathrm{a} \times \mathrm{b})$ effects on the gross motor development scores; the indirect effect and $84.73 \%$ of the total effect were mediated by the length of mother-infant separation. SARS-CoV-2 infection significantly increased the length of mother-infant separation (Path a, $p<0.001$ ), which significantly affected the gross motor development score (Path $\mathrm{b}, p=0.012$ ). The remaining direct effect of SARS-CoV-2 infection on the gross motor development score was not significant (Path $c^{\prime}, p=0.759$ ).

\section{DISCUSSION}

This is the first cohort study to evaluate the potential direct and indirect effects of SARS-CoV-2 infection during pregnancy (mainly in the third trimester) on early childhood development. We followed 57 mother-infant dyads in an infection cohort and 78 dyads in a non-infection cohort until 3 months after delivery. Our findings showed that SARS-CoV-2 infection during pregnancy did not increase the risks of social-emotional $(\mathrm{RR}=$ $0.87,95 \%$ CI: $0.51-1.49)$ and overall ( $R R=1.02,95 \%$ CI: $0.60-$ 1.73) developmental delay. However, the length of mother-infant separation was significantly negatively correlated with the gross motor score ( $\beta=-0.09,95 \%$ CI: -0.16 to -0.02$)$. Furthermore, using a mediation model, we found that SARS-CoV-2 infection had a significant indirect effect on the gross motor score by increasing the length of mother-infant separation.

Betacoronaviruses, including SARS-CoV, MERS-CoV, and SARS-CoV-2, have caused severe global pandemics. A study after the 2003 SARS epidemic showed that SARS-CoV infection during pregnancy did not increase the risk of developmental delay in children compared with children of mothers who were not infected with SARS-CoV during pregnancy (19). There was no evidence that MERS-CoV infection during pregnancy affected early offspring development. Current evidence suggests that SARS-CoV, MERS-CoV, and SARS-CoV-2 affect fewer children, cause fewer symptoms, and less severe disease, and are associated
TABLE 3 | Mediation effect between SARS-CoV-2 infection and score of gross motor domain of ASQ-3.

\begin{tabular}{|c|c|c|c|}
\hline Mediation path & $\beta(95 \% \mathrm{Cl})$ & $p$-value & $\begin{array}{l}\text { Proportion } \\
\text { mediated } \\
\left(a^{\star} b / c\right)\end{array}$ \\
\hline $\begin{array}{l}\text { COVID-19 effect on mediator } \\
(\text { Path a: } X \rightarrow M)\end{array}$ & $\begin{array}{c}34.14 \\
(26.90,41.38)\end{array}$ & $<0.001$ & $84.73 \%$ \\
\hline $\begin{array}{l}\text { Mediator effect on Gross } \\
\text { motor } \\
(\text { Path b: } M \rightarrow Y)\end{array}$ & $\begin{array}{c}-0.09 \\
(-0.16,-0.02)\end{array}$ & 0.012 & \\
\hline $\begin{array}{l}\text { Indirect effect } \\
\left(\text { Path } a^{*} b: X \rightarrow M\right)\end{array}$ & $\begin{array}{c}-3.14 \\
(-6.01,-0.20)\end{array}$ & - & \\
\hline $\begin{array}{l}\text { Direct effect } \\
\left(\text { Path } c^{\prime}: X \rightarrow Y \text { adjusted } M\right)\end{array}$ & $\begin{array}{c}-0.57 \\
(-4.21,3.08)\end{array}$ & 0.759 & \\
\hline $\begin{array}{l}\text { Total effect } \\
\text { (Path c: } X \rightarrow Y \text { adjusted } M)\end{array}$ & $\begin{array}{c}-3.71 \\
(-6.48,-0.93)\end{array}$ & 0.009 & \\
\hline
\end{tabular}

ASQ-3, Age and Stage Questionnaire Chinese version; Cl, confidence interval. $X$ independent variable (SARS-CoV-2 infection). $M$, mediator (mother-infant separation days). Y, dependent variable (score of gross motor development). Path a, the effect of SARS-CoV-2 infection on mediator. Path $b$, the effect of mediator on gross motor development. Path $a^{*} b$ (indirect effect), the mediated effect of SARS-CoV-2 infection on gross motor development by mother-infant separation days. Path $c^{\prime}$ (direct effect), the remaining effect of SARS-CoV-2 infection on gross motor development extent not explained by the mediators included in the model. Path c (total effect), the total effect of SARS-CoV-2 infection on gross motor development. In the mediation model, we adjusted maternal education (bachelor or above vs. high school or below), low birthweight (yes vs. no), infant gender (boy vs. girl), preterm (yes vs. no), admitted to NICU (yes vs. no), and breastfeeding at 3 months after birth (yes vs. no).

with much lower case-fatality rates, compared with adults (20, 21). Studies have proposed a potential mechanism of SARS-CoV2 infection during pregnancy on early offspring development, including maternal-fetal interface destruction, maternal systemic immune response, and direct brain infection interfering with brain development (22); however, these hypotheses have not been verified. Our findings provide epidemiological evidence that SARS-CoV-2 infection during pregnancy does not increase the risk of early developmental delay in offspring, compared with non-infected infants. In addition, it should be noted that the mothers in the infection cohort in the present study were mainly infected in the third trimester (accounting for 93.0\%). Our results cannot explain the impact on the neurodevelopment of offspring when the infection occurs in the early pregnancy, especially in the first trimester, which needs to be explained by more studies in the future.

In the further mediation model, we found that SARSCoV-2 infection during pregnancy had significant indirect effects on the ASQ-3 gross motor domain score by increasing the length of mother-infant separation. After separating the indirect effects, the remaining direct effect was not significant, which was consistent with the results of the multivariable linear regression models. To prevent neonatal infection (11), infants in the infection cohort experienced longer separation from their mothers than the non-infection cohort, which means that the infants in the infected group did not receive enough support and response from their mothers to meet their developmental needs (12). Early separation interrupted skinto-skin contact, breastfeeding, and mother-infant interactions, 


\begin{tabular}{l}
\hline SARS-CoV-2 infection \\
\cline { 3 - 4 }
\end{tabular}

which had adverse effects on the physiological development (23), innate and specific immunity (24), and neurodevelopment (including language, cognition, motor, and social development) of the children (25). In particular, we observed that the breastfeeding rate of the non-infected cohort at 1 and 3 months was $100 \%$, which was significantly higher than that of the infection cohort. Better breastfeeding would be more conducive to early childhood development. Although this study did not find its significant effect, it still deserves continuous attention. Thus, we confirmed the hypothesis of the mediation model that mother-infant separation may have a potential impact on early childhood development. Furthermore, our results also indicated that although the impact of SARS-CoV-2 infection during late pregnancy on early childhood development is limited, the secondary effects of the COVID-19 pandemic (such as motherinfant separation) may still affect early childhood development. It is also worth noting that a published study showed that when adequate protective measures are taken, the risk of infant infection caused by rooming-in practice is limited (26). Combined with our results, it is necessary to focus on the benefit of protected rooming-in, on the premise that the risk of infant infection can be effectively controlled.

The COVID-19 pandemic has caused both a health crisis and economic and social crises (27-29), which have profoundly affected sustainable development goals (30). The mother-infant separation discussed here is only the tip of the iceberg of this global crisis. The World Bank Group estimates that the global economy contracted $4.3 \%$ in 2020 ; the pandemic raised poverty rates to between 9.1 and $9.4 \%$, back to levels last seen in 2017 (31). Poverty is the source of many risk factors for early childhood development, including malnutrition, caregiver stress and depressive symptoms, low caregiver responsiveness, lowquality nurturing care, and community environment, causing them to miss early education (32). The COVID-19 pandemic is accelerating the deterioration of these external factors, pushing the poorest and most vulnerable people and their children into worse situations. We call for global attention to the plight of these families and their children in the current pandemic.

Our study had some limitations. First, the study population was small, since NERS reported only 138 pregnant women confirmed with COVID-19 as of April 30, 2020, which might affect the power of our study. Second, we found that only the length of mother-infant separation was negatively associated with the ASQ-3 gross motor domain score. Therefore, we used the gross motor domain score in the mediation model instead of the classification result. Therefore, we found only that SARS-CoV-2 infection had a potential indirect negative effect on the development of the children, which did not mean that this indirect effect would increase the risk of developmental delay in the offspring. In addition, the recruitment and follow-up were conducted through the internet and by telephone, and participant compliance was not as satisfactory as with face-to-face follow-up. Since our follow-up lasted only 3 months, our results cannot represent longer-term effects. Finally, we used screening scales instead of diagnostic scales to access early childhood development, which might cause evaluation bias.

In conclusion, we found that SARS-CoV-2 infection during late pregnancy did not increase the risk of offspring developmental delay at 3 months. However, we found that the length of mother-infant separation was significantly negatively 
correlated with the ASQ-3 gross motor domain score. We found that SARS-CoV-2 infection indirectly affected the gross motor score by increasing the length of mother-infant separation, mediating $84.73 \%$ of the total effect. Future researchers should conduct longer follow-up studies of children whose mothers were infected with SARS-CoV-2 during pregnancy, to evaluate the long-term effects of COVID-19 on early childhood development. Future researchers also need to pay more attention to potential mediators and indirect effects between COVID-19 and early childhood development as the pandemic is now changing the external environment of child development.

\section{DATA AVAILABILITY STATEMENT}

The raw data supporting the conclusions of this article will be made available by the authors, without undue reservation.

\section{ETHICS STATEMENT}

The studies involving human participants were reviewed and approved by Peking University Third Hospital Medical Science Research Ethics Committee (No. IRB00006761-M2020127). The patients/participants provided their written informed consent to participate in this study.

\section{REFERENCES}

1. World Health Organization. WHO Coronavirus Disease (COVID-19) Dashboard. (2021). Available online at: https://covid19.who.int/ (accessed July 07, 2021).

2. Di Mascio D, Khalil A, Saccone G, Rizzo G, Buca D, Liberati M, et al. Outcome of coronavirus spectrum infections (SARS, MERS, COVID-19) during pregnancy: a systematic review and meta-analysis. Am J Obstet Gynecol. (2020) 2:100107. doi: 10.1016/j.ajogmf.2020.100107

3. Allotey J, Stallings E, Bonet M, Yap M, Chatterjee S, Kew T, et al. Clinical manifestations, risk factors, and maternal and perinatal outcomes of coronavirus disease 2019 in pregnancy: living systematic review and metaanalysis. BMJ. (2020) 370:m3320. doi: 10.1136/bmj.m3320

4. Chen L, Li Q, Zheng D, Jiang H, Wei Y, Zou L, et al. Clinical characteristics of pregnant women with Covid-19 in Wuhan, China. N Engl J Med. (2020) 382:e100. doi: 10.1056/NEJMc2009226

5. Jafari M, Pormohammad A, Sheikh Neshin SA, Ghorbani S, Bose D, Alimohammadi S, et al. Clinical characteristics and outcomes of pregnant women with COVID-19 and comparison with control patients: a systematic review and meta-analysis. Rev Med Virol. (2021) 31:1-16. doi: $10.1002 / \mathrm{rmv} .2208$

6. Yu Y, Chen P. Coronavirus disease 2019 (COVID-19) in neonates and children from China: a review. Front Pediatr. (2020) 8:287. doi: $10.3389 /$ fped.2020.00287

7. Bloise E, Zhang J, Nakpu J, Hamada H, Dunk CE, Li S, et al. Expression of severe acute respiratory syndrome coronavirus 2 cell entry genes, angiotensinconverting enzyme 2 and transmembrane protease serine 2 , in the placenta across gestation and the maternal-fetal interface in pregnancies complicated by preterm birth or preeclampsia. Am J Obstetr Gynecol. (2021) 224:298.e1-e8. doi: 10.1016/j.ajog.2020.08.055

8. Yin M, Zhang L, Deng G, Han C, Shen M, Sun H, et al. Severe acute respiratory syndrome coronavirus 2 (SARS-CoV-2) infection during pregnancy in China: a retrospective cohort study. medRxiv [Preprint]. (2020). doi: 10.1101/2020.04.07.20053744

\section{AUTHOR CONTRIBUTIONS}

JQ, XW, YZ, and YWe conceived and designed the study. TW, LC, and YWa conducted the statistical analyses and drafted the initial manuscript. TW, LC, YWa, HS, JN, XY, ML, CT, HJ, and DZ conducted the follow-up surveys. HS and XW contributed to the interpretation of the data. All authors reviewed and revised the article. All authors read the final manuscript and approved submission.

\section{FUNDING}

This study was funded by the Chinese Academy of Engineering (2020-KYGG-01-06), the National Natural Science Foundation of China (72042013), the Peking University Health Science Center (BMU2020HKYZX001), and the National Key Research and Development Program of China (2016YFC1000307-2).

\section{ACKNOWLEDGMENTS}

We thank the National Health Commission of China for helping us to organize and coordinate this National Cohort Study. We also appreciate all investigators for their efforts in the follow-up process. We give special thank to all pregnant women and their families who participated in this study.

9. Rudolph MD, Graham AM, Feczko E, Miranda-Dominguez O, Rasmussen JM, Nardos R, et al. Maternal IL-6 during pregnancy can be estimated from newborn brain connectivity and predicts future working memory in offspring. Nat Neurosci. (2018) 21:765-72. doi: 10.1038/s41593-0180128-y

10. Rasmussen JM, Graham AM, Entringer S, Gilmore JH, Styner M, Fair DA, et al. Maternal Interleukin-6 concentration during pregnancy is associated with variation in frontolimbic white matter and cognitive development in early life. Neuroimage. (2019) 185:825-35. doi: 10.1016/j.neuroimage.2018.04.020

11. The Joint Prevention and Control Mechanism of the State Council of the People's Republic of China. Notice on Strengthening Maternal Disease Treatment and Safe Midwifery During the Prevention and Control of New Coronavirus Pneumonia. (2020). Available online at: http://www.gov.cn/ xinwen/2020-02/10/content_5476731.htm (accessed June 01, 2021).

12. Kacenelenbogen N, Dramaix-Wilmet M, Schetgen M, Roland M, Godin I. Parental separation: a risk for the psychomotor development of children aged 28 to 32 months? A cross-sectional study. BMC Pediatr. (2016) 16:89. doi: 10.1186/s12887-016-0621-y

13. Wang Y, Chen L, Wu T, Shi H, Li Q, Jiang H, et al. Impact of Covid-19 in pregnancy on mother's psychological status and infant's neurobehavioral development: a longitudinal cohort study in China. BMC Med. (2020) 18:347. doi: 10.1186/s12916-020-01825-1

14. National Health Commission and National Administration of Traditional Chinese Medicine. Diagnosis and treatment protocol for novel coronavirus pneumonia (Trial Version 7). Chin Med J. (2020) 133:1087-95. doi: 10.1097/CM9.0000000000000819

15. Yao GY, Bian XY, Squires J, Wei M, Song W. Cutoff scores of the ages and stages questionnaire-Chinese for screening infants and toddlers. Chin J Pediatr. (2010) 48:824-28. doi: 10.3760/cma.j.issn.0578-1310.2010.11.009

16. Bian XY, Xie HC, Squires J, Chen CY. Adapting a parentcompleted, socioemotional questionnaire in china: the ages \& stages questionnaires: social-emotional. Infant Ment Health J. (2017) 38:258-66. doi: 10.1002/imhj.21626 
17. Hayes F. Introduction to Mediation, Moderation, and Conditional Process Analysis: A Regression-Based Approach, 2nd Edn. New York, NY: Guilford Press (2017).

18. Qiao J. What are the risks of COVID-19 infection in pregnant women? Lancet. (2020) 395:760-2. doi: 10.1016/S0140-6736(20) 30365-2

19. Nasef N, O’Brien K, Wylie L, Unger S. Lessons from SARS: a retrospective study of outpatient care during an infectious disease outbreak. BMC Pediatr. (2010) 10:51. doi: 10.1186/1471-2431-10-51

20. Zimmermann P, Curtis N. Coronavirus infections in children including COVID-19: an overview of the epidemiology, clinical features, diagnosis, treatment and prevention options in children. Pediatr Infect Dis J. (2020) 39:355-68. doi: 10.1097/INF.00000000000 02660

21. Laws RL, Chancey RJ, Rabold EM, Chu VT, Lewis NM, Fajans M, et al. Symptoms and transmission of SARS-CoV-2 among children Utah and Wisconsin, March-May 2020. Pediatrics. (2021) 147:e2020027268. doi: $10.1542 /$ peds.2020-027268

22. López-Díaz Á, Ayesa-Arriola R, Crespo-Facorro B, Ruiz-Veguilla M. COVID19 infection during pregnancy and risk of neurodevelopmental disorders in offspring: time for collaborative research. Biol Psychiatry. (2020) 89:e29-e30. doi: 10.1016/j.biopsych.2020.09.011

23. Moore ER, Bergman N, Anderson GC, Medley N. Early skin-to-skin contact for mothers and their healthy newborn infants. Cochrane Database Syst Rev. (2016) 11:Cd003519. doi: 10.1002/14651858.CD0035 19.pub4

24. Cacho NT, Lawrence RM. Innate immunity and breast milk. Front Immunol. (2017) 8:584. doi: 10.3389/fimmu.2017.00584

25. Rocha N, Dos Santos Silva FP, Dos Santos MM, Dusing SC. Impact of mother-infant interaction on development during the first year of life: a systematic review. J Child Health Care. (2020) 24:365-85. doi: $10.1177 / 1367493519864742$

26. Ronchi A, Pietrasanta C, Zavattoni M, Saruggia M, Schena F, Sinelli MT, et al. Evaluation of rooming-in practice for neonates born to mothers with severe acute respiratory syndrome coronavirus 2 infection in Italy. JAMA Pediatrics. (2021) 175:260-6. doi: 10.1001/jamapediatrics.2020.5086
27. Wu T, Jia X, Shi H, Niu J, Yin X, Xie J, et al. Prevalence of mental health problems during the COVID-19 pandemic: a systematic review and metaanalysis. J Affect Disord. (2021) 281:91-8. doi: 10.1016/j.jad.2020.11.117

28. Jia X, Chen J, Li L, Jia N, Jiangtulu B, Xue T, et al. Modeling the prevalence of asymptomatic COVID-19 infections in the Chinese Mainland. Innovation. (2020) 1:100026. doi: 10.1016/j.xinn.2020.100026

29. World Bank Group. Poverty and Shared Prosperity. Washington, DC: World Bank Group (2020).

30. Heggen K, Sandset TJ, Engebretsen E. COVID-19 and sustainable development goals. Bull World Health Organ. (2020) 98:646. doi: 10.2471/BLT.20.263533

31. World Bank Group. Global Economic Prospects. Washington, DC: World Bank Group (2021).

32. Walker SP, Wachs TD, Grantham-McGregor S, Black MM, Nelson CA, Huffman SL, et al. Inequality in early childhood: risk and protective factors for early child development. Lancet. (2011) 378:1325-38. doi: 10.1016/S0140-6736(11)60555-2

Conflict of Interest: The authors declare that the research was conducted in the absence of any commercial or financial relationships that could be construed as a potential conflict of interest.

Publisher's Note: All claims expressed in this article are solely those of the authors and do not necessarily represent those of their affiliated organizations, or those of the publisher, the editors and the reviewers. Any product that may be evaluated in this article, or claim that may be made by its manufacturer, is not guaranteed or endorsed by the publisher.

Copyright (c) 2021 Wu, Chen, Wang, Shi, Niu, Yin, Li, Tan, Jiang, Zheng, Wei, Zhao, Wang and Qiao. This is an open-access article distributed under the terms of the Creative Commons Attribution License (CC BY). The use, distribution or reproduction in other forums is permitted, provided the original author(s) and the copyright owner(s) are credited and that the original publication in this journal is cited, in accordance with accepted academic practice. No use, distribution or reproduction is permitted which does not comply with these terms. 